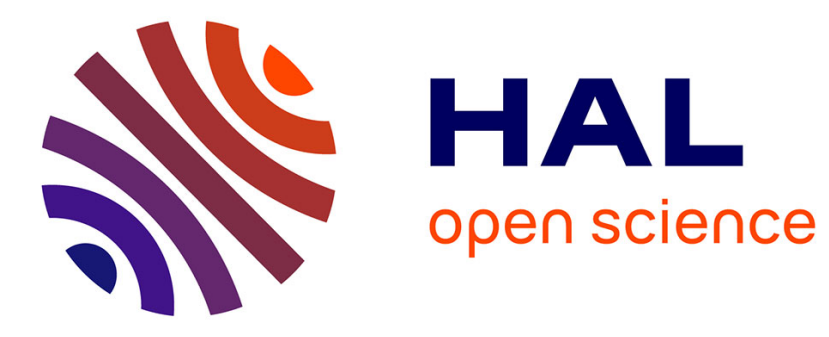

\title{
Impact of Sampling-Source Extinction Ratio in Linear Optical Sampling
}

Trung Hien Nguyen, Fausto Gomez Agis, Laurent Bramerie, Mathilde Gay, Jean-Claude Simon, Olivier Sentieys

\section{- To cite this version:}

Trung Hien Nguyen, Fausto Gomez Agis, Laurent Bramerie, Mathilde Gay, Jean-Claude Simon, et al.. Impact of Sampling-Source Extinction Ratio in Linear Optical Sampling. IEEE Photonics Technology Letters, 2013, 27 (7), pp.663-666. 10.1109/LPT.2013.2248353 . hal-00931661

\section{HAL Id: hal-00931661 \\ https://hal.inria.fr/hal-00931661}

Submitted on 15 Jan 2014

HAL is a multi-disciplinary open access archive for the deposit and dissemination of scientific research documents, whether they are published or not. The documents may come from teaching and research institutions in France or abroad, or from public or private research centers.
L'archive ouverte pluridisciplinaire HAL, est destinée au dépôt et à la diffusion de documents scientifiques de niveau recherche, publiés ou non, émanant des établissements d'enseignement et de recherche français ou étrangers, des laboratoires publics ou privés. 


\title{
Impact of Sampling-Source Extinction Ratio in Linear Optical Sampling
}

\author{
Trung-Hien Nguyen, Fausto Gomez-Agis, Laurent Bramerie, Mathilde Gay, \\ Jean-Claude Simon, and Olivier Sentieys
}

\begin{abstract}
We report on the impact of the sampling-source extinction ratio (ER) in a linear optical sampling system. To this effect, an analytical model is developed and contrasted with experimental data and numerical simulation, to determine the operational limits of the monitoring system. The ER of the reconstructed signal consists of a combined contribution from the magnitude of the symbol under test and the magnitude of the product between its neighboring symbols and the corresponding subpulses of the sampling source. The sampled signal can be completely reconstructed from its samples for ERs of the sampling source above $35 \mathrm{~dB}$.
\end{abstract}

Index Terms-Homodyne detection, optical fiber communications, optical pulse measurements, sampling methods.

\section{INTRODUCTION}

$\mathbf{T}$ HE monitoring of temporal waveforms in high-capacity optical communication networks has become an important requirement. Direct-detection, while being the most common and widespread method employed for this purpose, suffers from bandwidth limitations which restrict its use to monitoring serial-bit transmissions below $100 \mathrm{~Gb} / \mathrm{s}$. Various alternatives, such as nonlinear and linear-optical sampling whose temporal resolution can reach $700 \mathrm{GHz}$, have emerged to circumvent this physical limitation [1]. Nonlinear optical sampling is a detection technique, sensitive to optical intensity, that exploits $\chi^{2}$ and $\chi^{3}$ nonlinearities in semiconductors [2], planar waveguides [3], and highly nonlinear fibers [4]. However, the low sensitivity/efficiency inherent in the nonlinear optical mixing-processes requires large nonlinearities and high optical power. Moreover, phase-sensitive detection is possible with the aid of coherent detection, succeeding the nonlinear sampling [5]. On the other hand, linear-optical sampling is a coherent-like detection technique, which is sensitive to the electric field and yields information about both amplitude and phase of the sampled signal [signal under test (SUT)] [6]. The potential of linear-optical sampling for characterisation of temporal waveforms has been well documented in the

Manuscript received December 3, 2012; revised February 4, 2013; accepted February 15, 2013. Date of current version March 12, 2013. This work was supported in part by the ANR Project OCELOT under Grant ANR-2010VERS-015-03 and in part by the French Government.

T.-H. Nguyen, F. Gomez-Agis, L. Bramerie, M. Gay, and J.-C. Simon are with Université Européenne de Bretagne, Lannion 22305, France (e-mail: tnguyen@enssat.fr; fausto.gomez-agis@enssat.fr; laurent.bramerie@enssat.fr; mathilde.gay@enssat.fr; jean-claude.simon@enssat.fr).

O. Sentieys is with the INRIA/IRISA, Rennes 35000, France (e-mail: olivier.sentieys@irisa.fr).

Color versions of one or more of the figures in this letter are available online at http://ieeexplore.ieee.org.

Digital Object Identifier 10.1109/LPT.2013.2248353 literature, notably in terms of its sensitivity and high temporal resolution [6]-[8]. The principle of operation of this technique is based on the interference, in a $90^{\circ}$ optical-hybrid, of the SUT and a train of short pulses (the sampling signal). During the sampling instant, the electric field of the sampling pulse plays the role of local oscillator (LO) beating with the SUT in a linear detector [6], [7]. The sampled signal can then be reconstituted from the samples using signal-processing algorithms.

In [6] and [7], optical sampling was performed using low-repetition rate, passively mode-locked lasers $(10 \mathrm{MHz})$. Despite the low short-term jitter and phase noise, the longterm drift in the repetition rate of these lasers (linked to the uncontrolled temperature that modifies the cavity length) limits their use since the natural repetition rate then varies with time. Possible solutions to alleviate this constraint are either by using a pulse cavityless source which consists of two RF-driven stages (intensity and phase modulation) and an optimized length of dispersion-compensated fiber to produce pulses as short as 5 ps [9]; the pulses can then be subsequently compressed down to the sub-picosecond regime using an additional compression stage [10]. Or by employing actively mode-locked lasers, like monolithic lasers [11] or very-short external-cavity lasers [12] that produce pulses as short as 1 ps with highly-stable repetition frequency. Nevertheless, both solutions provide repetition frequencies $>2.5 \mathrm{GHz}$, which implies the use of expensive photodetectors (larger bandwidth) and analog-to-digital converters (ADCs) featuring at least 5 GS/s per channel. Therefore, solutions that loosen up the sampling rate of ADCs and reduce costs are of great concern.

In this letter, we investigate the possibility of using a train of pulses at high repetition rate laser, externally intensity modulated to produce a lower repetition rate, as the sampling signal source for linear optical sampling. Although this situation enables the use of affordable ADCs with sampling rates below $2 \mathrm{GS} / \mathrm{s}$ per channel together with the use of highly-stable repetition-frequency pulsed-laser, the question of accuracy of reconstructing the SUT with a LO limited in extinction ratio (ER) arises because of limited ER of high-frequency modulators. In order to simplify an analytical analysis, the particular case of synchronous and homodyne signals is considered followed by a comparison with numerical and experimental data. The more general case, intradyne and asynchronous signals, will be addressed in future work. Additional requirements of the sampling source in a linear optical sampling system, such as pulsewidth, chirp, detuning frequency, etc. are critical and were fully studied in [13]. 


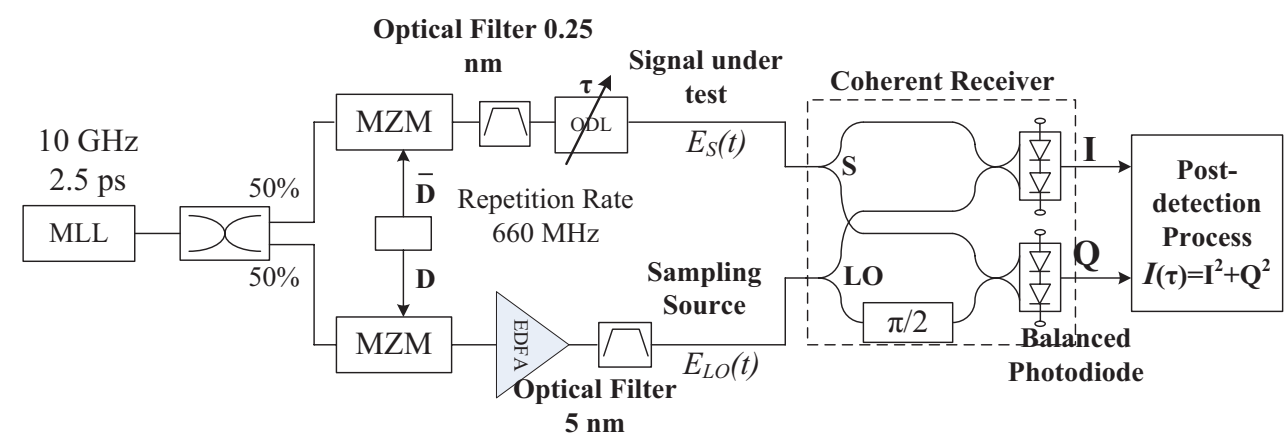

Fig. 1. Experimental setup for modeling and characterizing the impact of $E R_{L O}$ in linear optical sampling.

This letter is organized as follows. Section II presents an analytical description of the impact of ER in linear optical sampling. In section III, the experimental verification of the analytical model is exposed. Discussion and comparison of experimental and analytical versus numerical results are given in section IV. Finally, conclusions are drawn in section V.

\section{Analytical Model}

\section{A. Linear Optical Sampling}

The concept of linear optical sampling is schematically depicted in Fig. 1 (dashed square line). The data signal $E_{S}(t)$ and a train of sampling pulses $E_{L O}(t)$ are coupled into a $90^{\circ}$ optical hybrid-unit, where they are split and recombined, producing two interference terms: the in-phase and quadrature at the ports I and $\mathrm{Q}$, respectively. The $90^{\circ}$ phase change in the lower branch of the splitting coupler, in the optical hybrid, produces the quadrature interference term, whereas the upper coupler produces the in-phase interference term. Next, balanced photodetection is realized, followed by postprocessing of the acquired samples. Following the formalism and assumptions employed in [8], the intensity of the reconstructed signal, including the filtering effects of the bandwidthlimited receiver, is expressed as

$$
I(t, \tau)=16\left|\left[E_{S}(t) E_{L O}^{*}(t-\tau)\right] \otimes h(t)\right|^{2}
$$

where $h(t)=f_{c} \operatorname{sinc}\left(f_{c} t\right)$ is the impulse response of the balanced receiver with $f_{c}$ being the $3-\mathrm{dB}$ bandwidth. The symbol $\otimes$ and $*$ represents the convolution and complexconjugated operations, respectively.

\section{B. Case Study}

In order to study the impact of distortions of the pulsed LO, such as pedestals, ghost pulses, or sub-pulses due to the external modulation, on the sampling and reconstruction process of the SUT, due to the limited ER of the external modulation, the following conditions are considered based on the diagram shown in Fig. 1. Here, the SUT and the sampling pulses train are perfectly synchronous and carried by the same wavelength (homodyne condition). Both signals have steady normalized amplitudes, the same repetition frequency and the same pulse shape; noise effects will be ignored. The SUT will be represented by a stream of large pulses featuring a periodic low level with a fixed ER, the value of which will depend on the optical switch (e.g. an intensity modulator) used to convey the low level (See Fig. 2). The sampling signal will be represented by an inverted version of the SUT but featuring a shorter pulse duration, and will be designated by the Dirac $\delta$-function $\delta(t)$.

The corresponding amplitudes for the interacting signals are defined as

$$
\begin{aligned}
& \left|E_{S}(t)\right| \\
& =\sum_{n=-\infty}^{\infty}\left[\sqrt{\frac{P}{E R_{S}}} f\left(t-n T_{S}\right)+\sum_{l_{1}=1}^{k-1} \sqrt{P} f\left(t-n T_{S}-l_{1} T\right)\right] \\
& \left|E_{L O}(t)\right| \\
& =\sum_{m=-\infty}^{\infty}\left[\delta\left(t-m T_{S}\right)+\sqrt{\frac{1}{E R_{L O}}} \sum_{l_{2}=1}^{k-1} \delta\left(t-m T_{S}-l_{2} T\right)\right]
\end{aligned}
$$

where $f(t)$ defines the pulse-shape of the amplitude envelope $\left|E_{S}(t)\right|$, indices $m$ and $n$ indicate the number of periods $T_{S}$ in the sampling train and the SUT respectively while $T$ defines the pulse time-slot. $E R_{S}$ and $E R_{L O}$ indicate the respective ERs of the SUT and the sampling pulse train. $P$ is the peak power of the SUT and $k=T_{S} / T$ gives the total number of pulses within one period for $E_{S}$ and $E_{L O}$.

For this analysis, it is assumed that $f(t)=\operatorname{sech}^{2}\left(t / T_{1}\right)$ with $T_{1}$ as the full-width at the $1 / e$ maximum of the intensity profile. By using (1), (2) and (3), the intensity at the sampling instant $\tau$ of the low level can be expressed as

$$
\begin{gathered}
I^{0}(t, \tau)=\frac{P}{E R_{S} T_{c}^{2}} \operatorname{sech}^{2}\left(\frac{\tau}{T_{1}}\right) \operatorname{sinc}^{2}\left(\frac{t}{T_{c}}-\frac{\tau}{T_{c}}\right) \\
+\sum_{l=1}^{k-1} \frac{P}{E R_{L O} T_{c}^{2}} \operatorname{sech}^{2}(t-l T-\tau) \operatorname{sinc}^{2}\left(\frac{t}{T_{c}}-\frac{l}{\left(T_{c} / T\right)}-\frac{\tau}{T_{c}}\right)
\end{gathered}
$$

where $T_{c}=1 / f_{c}$ and $f_{c}$ is the receiver bandwidth. $m=n=0$ indicates for one period of $E_{S}$ and $E_{L O}$. The first term on the right-hand side of (4) represents the magnitude of the symbol under test while the second represents an additional contribution consisting of the product of neighboring symbols from the SUT and the corresponding sub-pulses of the pulsed LO. It is seen that the signal after detection is modified due 

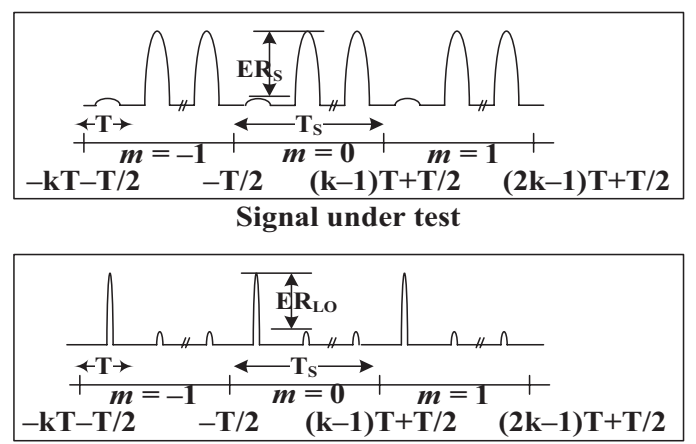

Sampling Source

Fig. 2. Temporal traces for the signal under test and the sampling signal.

to the impulse-response of the receiver bandwidth $f_{c}$, which defines the total number of sub-pulses contributing at the current sampling instant as $k_{n}-1=\left(T_{c}-T\right) / T$.

Finally, the normalized intensity of the reconstituted SUT $\overline{I(\tau)}$ is given by (5). The first term represents the low level of the SUT, while the second term represents the high level. It is worthwhile to point out that there are two main contributions to $\overline{I(\tau)}$ : a) the ER of the symbol under test; and b) the product between neighboring symbols of the SUT and the ER of the sampling signal $E R_{L O}$.

$\overline{I(\tau)}=\left\{\begin{array}{c}{\left[\frac{1}{E R_{S}}+\frac{1}{E R_{L O}}\left(k_{n}-1\right)\right] \operatorname{sech}^{2}\left(\frac{\tau}{T_{1}}\right),} \\ \text { if } \tau \in\left[-\frac{T}{2}, \frac{T}{2}\right) \\ \sum_{l=1}^{k-1}\left[\frac{1}{E R_{S} E R_{L O}}+1+\frac{1}{E R_{L O}}\left(k_{n}-2\right)\right] \operatorname{sech}^{2}\left(\frac{\tau-l T}{T_{1}}\right), \\ \text { if } \tau \in\left[\frac{T}{2}, T_{S}\right]\end{array}\right.$

The achievable ER of the reconstructed signal $E R_{\text {out }}$, can be specified as a figure of merit for the sampling system. It is defined as the ratio of the high level to the low level, represented in (5) at $\tau=T$ and $\tau=0$, respectively, and is given by

$$
E R_{\text {out }}=\frac{1+E R_{S} E R_{L O}+\left(k_{n}-2\right) E R_{S}}{E R_{L O}+\left(k_{n}-1\right) E R_{S}}
$$

clearly revealing the impact of $E R_{L O}$ in the reconstruction of the SUT.

\section{EXPERIMENT}

The experimental setup for the study of the ER-impact of the sampling source in the linear sampling process is shown in Fig. 1. Optical pulses, generated by a mode-locked laser at $1550 \mathrm{~nm}$ with pulse duration of $2.5 \mathrm{ps}$ and $10 \mathrm{GHz}$ repetition rate, are split via an optical 50-50 coupler to form the SUT and the sampling signal. The repetition frequency of the sampling signal is reduced down to $660 \mathrm{MHz}$ by means of external modulation, with one 1 for each fifteen 0's. Next, the sampling signal is amplified and filtered by an optical band-pass filter (OBPF) with 3-dB bandwidth of $5 \mathrm{~nm}$, and coupled to the LO port of the $90^{\circ}$ optical-hybrid. Since we are interested in studying the impact of the $E R_{L O}$ on the sampled low level of the SUT which will feature one 0 for fifteen 1's; namely an

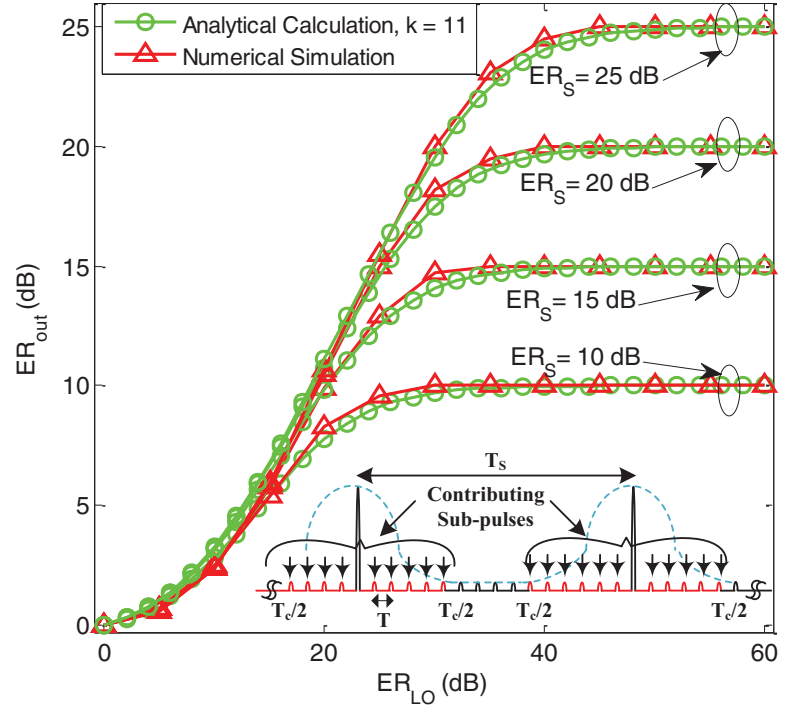

Fig. 3. Relationship between ERLO and ERout for the different values of ERS.

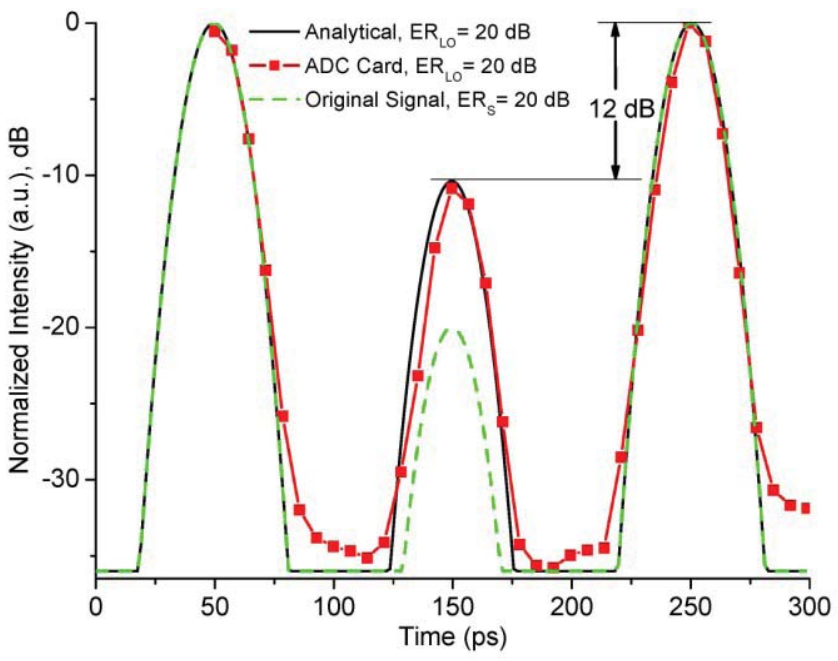

Fig. 4. Analytical prediction of $\overline{I(\tau)}$, with ERLO $=20 \mathrm{~dB}$, validated by experimental data.

inverted copy of the sampling signal. The SUT is amplified and filtered by an OBPF with $3-\mathrm{dB}$ bandwidth of $0.25 \mathrm{~nm}$, resulting in a pulse duration of 25 ps. Subsequently, the SUT is coupled into the $\mathbf{S}$ port of the hybrid-unit. The ER for the SUT and sampling signal, $E R_{S}$ and $E R_{L O}$, are both around $20 \mathrm{~dB}$. The average optical power for both signals at the input of the hybrid-unit is of $-10 \mathrm{dBm}$ and the hybrid-unit losses are of the order of $11 \mathrm{~dB}$. Since the two signals are initially synchronized, a variable optical delay is needed to allow scanning across the symbol under test. This is done by placing a tunable optical-delay line (ODL) in the SUT path. The in-phase and quadrature output terms at the I and $\mathbf{Q}$ ports, respectively, are then detected by means of two balanced photodetectors with electrical bandwidth of $800 \mathrm{MHz}\left(T_{c}=\right.$ $1 / 800 \mathrm{MHz}$ ). Next, the respective electrical signals are sampled by an ADC card and followed by post-processing. 


\section{RESUlTS AND Discussion}

To understand the impact of $E R_{L O}$ in the reconstruction of the sampled signal, $E R_{\text {out }}$ is evaluated from (6) using the following parameters: $E R_{S}$ is changed from 10 to $25 \mathrm{~dB}$ in steps of $5 \mathrm{~dB}$, while $E R_{L O}$ varies from 0 to $60 \mathrm{~dB}$. $\left(k_{n}-1\right)$, representing the number of sub-pulses contributing to the sample instant of the symbol under test, as schematically shown in the inset of Fig. 3, is estimated at 11 from $k_{n}-1=\left(T_{c}-T\right) / T=1.1 \mathrm{~ns} / 0.1 \mathrm{~ns}$. In other words, the contributing sub-pulses fall within the electrical detection bandwidth shown as the dashed line in the inset of Fig. 3. To validate this statement, a numerical simulation including the effect of the photodetection bandwidth has been carried out. The relationship between $E R_{S}$ and $E R_{L O}$ is plotted in Fig. 3, where the circles represent the analytical calculation and the triangles the numerical simulation. Numerical simulation and analytical calculation reveal negligible differences. From the figure, it can be observed that the sampled signal is completely reconstructed when $E R_{L O}$ goes above $35 \mathrm{~dB}$, whereas for lower values, a less accurate representation of the SUT is obtained. For $E R_{S}$ below $10 \mathrm{~dB}$, the $E R_{L O}$ requirement relaxes to about $20 \mathrm{~dB}$. The stringent requirements for $E R_{L O}$ are due to the additional contribution to the sampled symbol, which comes from the interaction between neighboring symbols and the corresponding sub-pulses of the sampling signal with magnitude $1 / E R_{L O}$ (as shown in the inset of Fig. 3). The intensity of the reconstructed signal for the sequence of 1 , 0,1 , as expressed by (5), is represented in Fig. 4 for $E R_{L O}$ equal to $20 \mathrm{~dB}$. Also in the figure, experimental data of the sampled sequence have been included for comparison. The dash-line represents the analytical calculation of SUT with the assumption of a noise floor at about $-35 \mathrm{~dB}$, whereas the continuous line corresponds to the analytical calculation of the sampled and reconstructed SUT. Experimental data is represented by square symbols. Compared with experimental results, numerical simulations indicate that $E R_{\text {out }}$ can be predicted accurately. For the experiment carried out, an $E R_{\text {out }}$ of $12 \mathrm{~dB}$ was determined. The difference in noise floor between the experimental measurements and the theoretical calculations is due to the limited dynamic-range of the ADC card used to sample the electrical signals coming from the balancedphotodetector.

\section{CONCLUSion}

In this letter, we report on our investigation of the impact of the sampling-source extinction ratio (ER) in a linear optical sampling system. This study was carried out by analytical calculations and validated by numerical simulations and experiments. The ER of the reconstructed signal is a combined contribution from the magnitude of the symbol under test and the magnitude of the product between its neighboring symbols and the corresponding sub-pulses of the pulsed local oscillator. The signals to be characterized can be completely reconstituted from their samples, when $E R_{L O}$ is above $35 \mathrm{~dB}$. Likewise, for $E R_{S}$ below $10 \mathrm{~dB}$, the $E R_{L O}$ requirement relaxes to about $20 \mathrm{~dB}$. This analytical model therefore represents a useful and simple tool for predicting the operational limits of a linearoptical sampling in terms of ER.

\section{REFERENCES}

[1] S. Watanabe, et al., "Novel fiber Kerr-switch with parametric gain: Demonstration of optical demultiplexing and sampling up to $640 \mathrm{~Gb} / \mathrm{s}$,' in Proc. 30th ECOC 2004, Sep., pp. 1-27, paper Th4.1.6.

[2] P. J. Maguire, et al., "Highly-efficient optical sampling based on twophoton absorption in a semiconductor micro-cavity device," in Proc. CLEO, May 2005, pp. 1-3, paper CTuAA5.

[3] J. V. Erps, et al., "High-resolution optical sampling of 640-Gb/s data using four-wave mixing in dispersion-engineered highly nonlinear $\mathrm{As}_{2} \mathrm{~S}_{3}$ planar waveguides," J. Lightw. Technol., vol. 28, no. 2, pp. 209-215, Jan. 15, 2010.

[4] P. A. Andrekson, "Picosecond optical sampling using four-wave mixing in a fiber,' Electron. Lett., vol. 27, no. 16, pp. 1440-1441, Aug. 1991.

[5] H. Sunnerud, M. Westlund, M. Sköld, and P. A. Andrekson, "All-optical balanced detection system with sub-ps resolution," in Proc. Opt. Fiber Commun. Conf., Mar. 2009, pp. 1-3, paper OThF4.

[6] C. Dorrer, C. R. Doerr, I. Kang, R. Ryf, J. Leuthold, and P. J. Winzer, "Measurement of eye diagrams and constellation diagrams of optical sources using linear optics and waveguide technology," J. Lightw. Technol., vol. 23, no. 1, pp. 178-186, Jan. 2005.

[7] C. Dorrer, D. C. Kipler, H. R. Stuart, and G. Raybon, "Ultra-sensitive optical sampling by coherent-linear detection," in Proc. Opt. Fiber Commun. Conf., Mar. 2002, pp. FD5-1-FD5-3, paper FD5.

[8] C. Dorrer, D. C. Kipler, H. R. Stuart, G. Raybon, and M. G. Raymer, "Linear optical sampling," IEEE Photon. Technol. Lett., vol. 15, no. 12, pp. 1746-1748, Dec. 2003.

[9] J. J. Veselka and S. K. Koroty, "Pulse generation for soliton systems using lithium niobate modulators," IEEE J. Sel. Topics Quantum Electron., vol. 2, no. 2, pp. 300-310, Jun. 1996.

[10] H. Hu, et al., "10 GHz pulse source for $640 \mathrm{Gbit} / \mathrm{s}$ OTDM based on $\mathrm{LiNbO}_{3}$ modulators and self-phase modulation," in Proc. ECOC 2011, Sep., pp. 1-3, paper We.10.P1.44.

[11] F. Lelarge, et al., "Recent advances on InAs/InP quantum dash based semiconductor lasers and optical amplifiers operating at $1.55 \mu \mathrm{m}$,' IEEE J. Sel. Topics Quantum Electron., vol. 13, no. 1, pp. 111-124, Jan./Feb. 2007.

[12] R. Ludwig and A. Ehrhardt, "Turn-key-ready wavelength-, repetition rate- and pulsewidth-tunable femtosecond hybrid modelocked semiconductor laser,' Electron. Lett., vol. 31, no. 14, pp. 1165-1167, Jul. 1995.

[13] I. Kim, C. Kim, and G. Li, "Requirements for the sampling source in coherent linear sampling," Opt. Express, vol. 12, no. 12, pp. 2723-2730, Jun. 2004 